Office of Naval Research

Contract Nonr-1866 (16) NR-372-012

NATIONAL AERONAUTICS AND SPACE ADMINISTRATION

Grant NGR 22-007-068

\title{
THE SEPARATE COMPUTATION OF ARCS FOR OPTIMAL FLIGHT PATHS WITH STATE VARIABLE INEQUALITY CONSTRAINTS
}

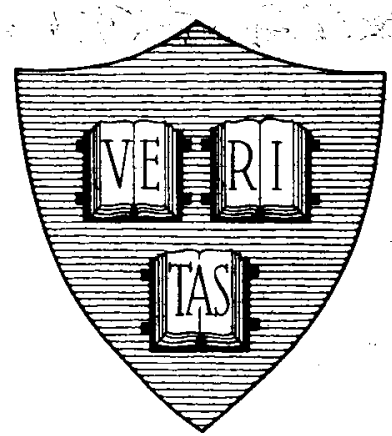

By

Jason L. Speyer, Raman K. Mehra and Arthur E. Bryson, Jr.

May 1967

Technical Report No. 526

"Reproduction in whole or in part is permitted by the U. $S$.

Government. Distribution of this document is unlimited. ".

Division of Engineering and Applied Physics

Harvard University - Cambridge. Massachusetts 
Office of Naval Research

Contract Nonr $-1866(16)$

NR - $372-012$

National Aeronautics and Space Administration

Grant NGR-22-007-068

THE SEPARATE COMPUTATION OF ARCS FOR

OPTIMAL FLIGHT PATHS WITH STATE VARIABLE

INEQUALITY CONSTRAINTS

By

Jason L. Speyer, Raman K. Mehra, and Arthur E. Bryson, Jr.

Technical Report No. 526

Reproduction in whole or in part is permitted by the U. S.

Government. Distribution of this document is unlimited.

May 1967

The research reported in this document was made possible through support extended the Division of Engineering and Applied Physics, Harvard University by the U.S. Army Research Office, the U. S. Air Force Office of Scientific Research and the U. S. Office of Naval Research under the Joint Services Electronics Program by Contracts Nonr-1866(16), (07), and (32), and NASA Grant NGR-22-007068 .

Division of Engineering and Applied Physics

Harvard University Cambridge, Massachusetts 


\title{
* the separate computation of arcs for optimal
}

\section{FLIGHT PATHS WITH STATE VARIABLE}

INEQUALITY CONSTRAINTS

By

Jason L. Speyer, Raman K. Mehra, and Arthur E. Bryson, Jr.

Division of Engineering and Applied Physics

Harvard University Cambridge, Massachusetts

\begin{abstract}
A BSTRACT
Separate computation of arcs is possible for a large class of optimization problems with state variable inequality constraints. Surprisingly, this class (to the best of the authors' knowlege) includes all physical problems which have been solved analytically or numerically to date. Typically these problems have only one constrained arc. Even in more complex problems, separation of arcs can be used to search for additional constrained arcs.
\end{abstract}

As an important example, a maximum range trajectory for a glider entering the Earth's atmosphere at a supercircular velocity is determined, subject to a maximum altitude constraint after initial pull-up. It is shown that the optimal path can be divided into three arcs, which may be determined separately with no approximations. The three arcs are (1) the initial arc, beginning at specified initial condition and ending at the entry point onto the altitude constraint; (2) the arc lying on the altitude constraint; and (3)the terminal arc, beginning at the exit point of the altitude constraint and ending at some specified terminal altitude.

* The work reported was partially supported by the Space and Information Systems Division of the Raytheon Company. 


\section{A BSTRACT (Cont'd)}

The conjugate gradient method, (ref. 4), a first order optimization scheme, is shown to converge very rapidly to the individual unconstrained optimal arcs. Using this optimization scheme and taking advantage of the separation of arcs an investigation revealed that two locally optimum paths exist. The range of one exceeds the range of the other by about 250 nautical miles (about 6\%) for the re-entry vehicle used here (maximum lift-to-drag ratio is .9). 


\section{INTRODUCTION}

In the past few years techniques for solving optimal programming problems with a state variable inequality constraint (SVIC) have been developed. Necessary conditions for a stationary solution were given by Gamkrelidze [1], and Bryson, Denham, and Dreyfus, [2] . One numerical technique for solving such problems uses a "penalty function" which requires the introduction of an auxiliary state variable [3], [4]. An improvement over the "penalty function" method, in both speed and accuracy, is the direct approach [5], where the SVIC is satisfied without using an extra state variable. In both techniques, the equations of motion and the EulerLagrange equations must be integrated over the entire path for each iteration.

The present paper shows that for certain problems with a SVIC, the computation of the state and Euler-Lagrange variables need only be done on the unconstrained arcs. Numerical computation of shorter unconstrained paths allows more rapid convergence and increased numerical accuracy. Also, if the constrained arc forms a large part of the entire path, this 
greatly reduces the amount of computation required. This separation of arcs occurs, for example, in the problem of finding the maximum range of a glider entering the Earth's atmosphere at parabolic velocities subject to a maximum altitude constraint after initial pull-up (sketch of possible trajectory in altitude-range space is shown in Fig. 1). This problem was solved by the direct method of reference 5 and by the penalty function method in reference 11 . The independence of the unconstrained arcs can be seen by observing that on the constant altitude constraint two of the three state variables are fixed (altitude and flight path angle); the velocity decreases due to the drag force. Velocity vs. range is a universal curve on this arc; only the velocity at the beginning and the end of this arc need be determined. A maximum range path, starting at any velocity on the constraint boundary that is higher than the velocity at the end of the constrained arc, has the same unconstrained path from the exit point of the altitude constraint to the terminal altitude. Similarly, a maximum range path, ending at any velocity on the constraint boundary that is lower than the velocity at the beginning of the constrained arc, has the same unconstrained path from the inital point to the entry point onto the constraint boundary. The unconstrained arcs can be found separately, determining the velocities at the beginning and the end of the constrained arc in the process. Having these velocities, the range on the altitude constraint can be easily evaluated. The three arcs put together form the maximizing path, without any approximations.

Such separation of arcs is possible if the number of variables on which the motion and constraints depend explicitly is larger by one than the order of the SVIC. The order of a SVIC is defined as the number of 


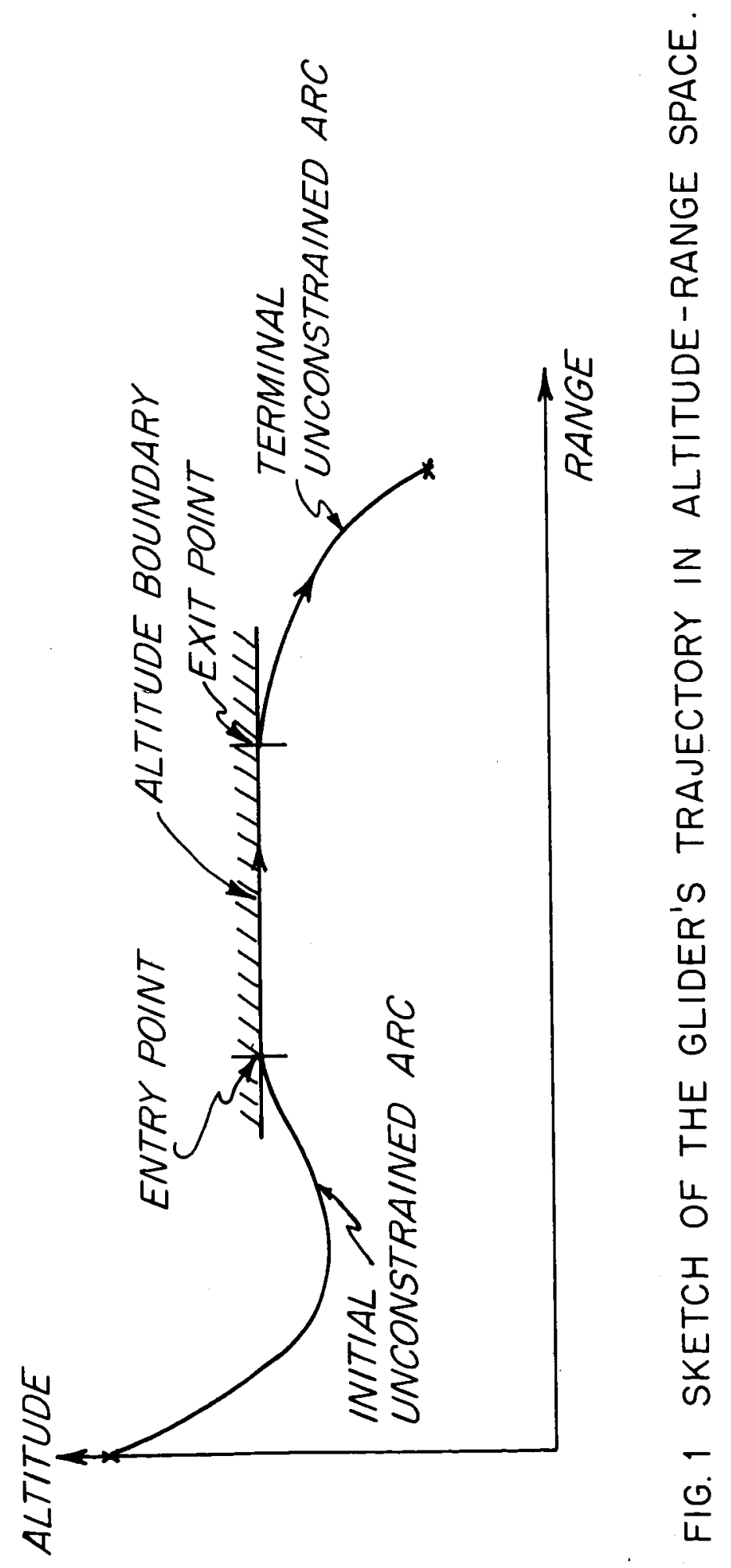


differentiations of the SVIC function needed for the control variable to appear explicitly (cf. ref. 2)

\section{PROBLEM FORMULATION}

The general problem considered here is to determine a control program $u(t)$, in the interval $t_{0} \leqq t \leqq t_{f}$ so as to maximize

$$
J=\int_{t}^{t_{f}} g(x, u, t) d t
$$

subject to the constraints

$$
\begin{aligned}
& \dot{x}=f(x, u, t) \\
& M=M\left[x\left(t_{f}\right), t_{f}\right] \\
& S(x, t) \leqslant 0 \\
& t_{o} \text { and } x\left(t_{o}\right) \text { given }
\end{aligned}
$$

where $t$ (time) is the independent variable; $(\cdot)$ is $d / d t(l) ; u(t)$ is a scalar control variable; $x(t)$ is an $n$-vector of state variables; $f$ is an $n$ vector of known functions of $x(t), u(t)$, and $t$, and is assumed everywhere differentiable with respect to $x$ and $u, M$ is a $q$-vector of known functions of $x\left(t_{f}\right)$ and $t_{f}, q \leqslant n ; S$ is a scalar function of $x(t)$ and $t$.

For those intervals of time that an extremal solution lies on a $p^{\text {th }}$ order SVIC boundary $(S(x, t)=0)$ it is necessary that $S$ and all its time derivatives that do not contain the control be zero:

$$
\left[\mathrm{s}, \dot{\mathrm{s}}, \ldots, \mathrm{s}^{(\mathrm{p}-1)}\right]^{\mathrm{T}}=0
$$


The value of the control which keeps (6) satisfied along the constrained path is obtained by the $p^{\text {th }}$ derivative of $S$

$$
S^{(p)}(x, u, t)=0
$$

It is assumed that the control on the constraint boundary can be found as a function of $(x, t)$ from the implicit equation (7) in the form

$$
u=\Lambda(x, t)
$$

\section{SUFFICIENT CONDITIONS FOR SEPARATE COMPUTATION OF ARCS}

Separation of arcs is possible if the contribution of the constrained arc to the performance index depends only on the entry and exit values of one variable ( $t$ or some element of $x$ ). Suppose the contribution of the constrained arc to the performance index, $J\left(t_{1}, t_{2}\right)$ is

$$
J\left[t_{1}, t_{2}\right]=\int_{t_{1}}^{t_{2}} g(x, u, t) d t
$$

where $t_{1}$ is the entry point time and $t_{2}$ is the exit point time. If $p=n$ then (6) can be used to solve for all the variables in terms of one, say $x_{1}$. Let the remaining $n-1$ state variables be denoted by the vector $y$. Then from (6)

$$
\left[\begin{array}{l}
y \\
t
\end{array}\right]=\mathbf{r}\left(x_{1}\right)
$$

All the variables in (9) can be eliminated except $x_{1}$ if $(y, t, u)$ are eliminated using (10) and (8) and the variable of integration is changed from $t$ to $x_{1}$ by the differential element of $x_{1}$ in (2) as

$$
x_{1}=f_{1}\left(x_{1}, y, t, u\right)
$$


Thus (9) becomes

$$
\begin{aligned}
J\left[t_{1}, t_{2}\right] & =\int_{x_{1}\left(t_{1}\right)}^{x_{1}\left(t_{2}\right)} \frac{g\left\{x_{1}, r\left[x_{1}\right], \Lambda\left[x_{1}, r\left(x_{1}\right)\right]\right\}}{f_{1}\left\{x_{1}, r\left(x_{1}\right), \Lambda\left[x_{1}, r\left(x_{1}\right)\right]\right\}} d x_{1} \\
& =\int_{x_{1}\left(t_{1}\right)}^{x_{1}\left(t_{2}\right)} G\left(x_{1}\right) d x_{1}=K\left[x_{1}\left(t_{2}\right)\right]-K\left[x_{1}\left(t_{1}\right)\right]
\end{aligned}
$$

It is tacitly assumed that starting from any value of $x_{1}\left(t_{1}\right)$ on the constrained arc, the value of $x_{1}\left(t_{2}\right)$ will eventually be reached.

If (12) is possible then the optimization problem can be separated into two smaller optimization problems. They are; find $u(t)$ to maximize

$$
J_{1}=J\left[t_{0}, t_{1}\right]-K\left[x_{1}\left(t_{1}\right)\right]
$$

subject to $(2),(5)$ and the corner conditions of (6) and; find $u(t)$ to maximize

$$
J_{2}=J\left[t_{2}, t_{f}\right]+K\left[x_{1}\left(t_{2}\right)\right]
$$

subject to (2), (3) and the initial conditions of (6). The sum of (13) and (14) will give the maximum value of (1).

If the equations of motion and boundary conditions do not explicity depend upon clock time but only on time elapsed from the initial time, then the arcs will separate for $n-1=p$.

4. MAXIMUM RANGE OF A HYPERSONIC GLIDER WITH AN ALTITUDE CONSTRAINT

The ideas of the previous section are applied here to the problem of 
$-7-$

maximizing the range of a glider (entering the Earth's atmosphere* at parabolic speeds) with an inflight constraint on the maximum altitude after pull-up. This problem, originally thought to be a complicated problem with a SVIC (ref. 5), falls into the special class of separable problems.

The nomenclature for this problem is given in Fig. 2. The aerodynamic forces, lift and drag, are varied through the control variable $\alpha(t)=$ angle-of-attack. The lift-drag characteristics of the glider are shown in Fig. 3. The wing loading of the glider $\mathrm{mg} / \mathrm{S}$, was taken as $61.31 \mathrm{~b} . \mathrm{ft}^{-2}$. The 1956 ARDC standard atmosphere model was used. The glider is approximated as a point mass moving about a spherical nonrotating Earth. The equations of motion are:

$$
\begin{aligned}
& \dot{\mathrm{V}}=\frac{-\mathrm{C}_{D} \rho \mathrm{V}^{2} \mathrm{~S}}{2 \mathrm{~m}}-\mathrm{g} \sin \gamma \\
& \dot{Y}=\frac{\mathrm{C}_{L} \rho \mathrm{VS}}{2 \mathrm{~m}}+\left(\frac{\mathrm{V}}{\mathrm{R}+\mathrm{h}}-\frac{\mathrm{g}}{\mathrm{V}}\right) \cos Y \\
& \dot{\mathrm{h}}=\mathrm{V} \sin \gamma
\end{aligned}
$$

The problem is to find the control program, $\alpha(t)$, which maximizes the range

$$
R_{A}=\int_{t_{0}}^{t_{f}} \frac{V}{1+h / R} \cos Y d t
$$

subject to (15),(16), and (17), with initial conditions on $V, Y$, and $h$, and a

\footnotetext{
* Actually the problem is started in the Earth's atmosphere partly to save computer time and partly because the control force is negligible compared to the centrifugal force during most of the omitted path.
} 


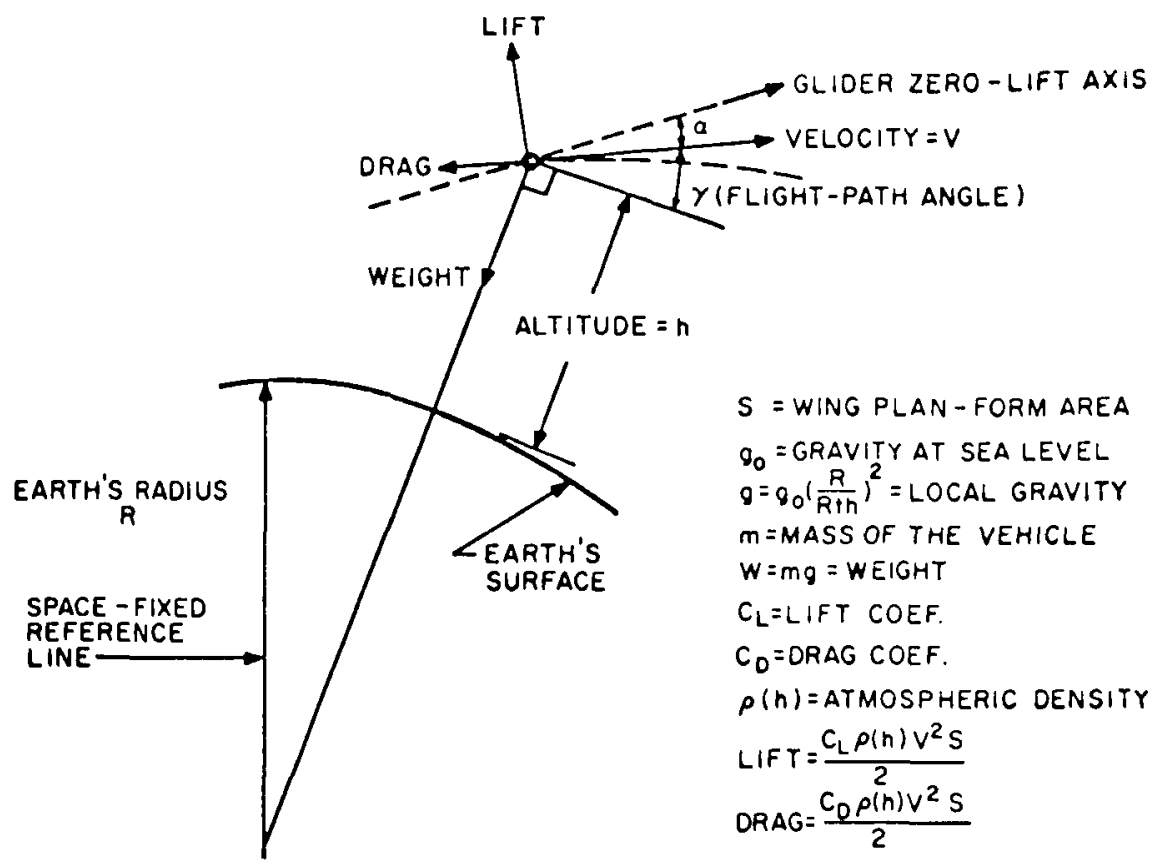

FIG. 2 GEOMETRY AND NOMENCLATURE OF ATMOSPHERIC RE-ENTRY EXAMPLE PROBLEM. 


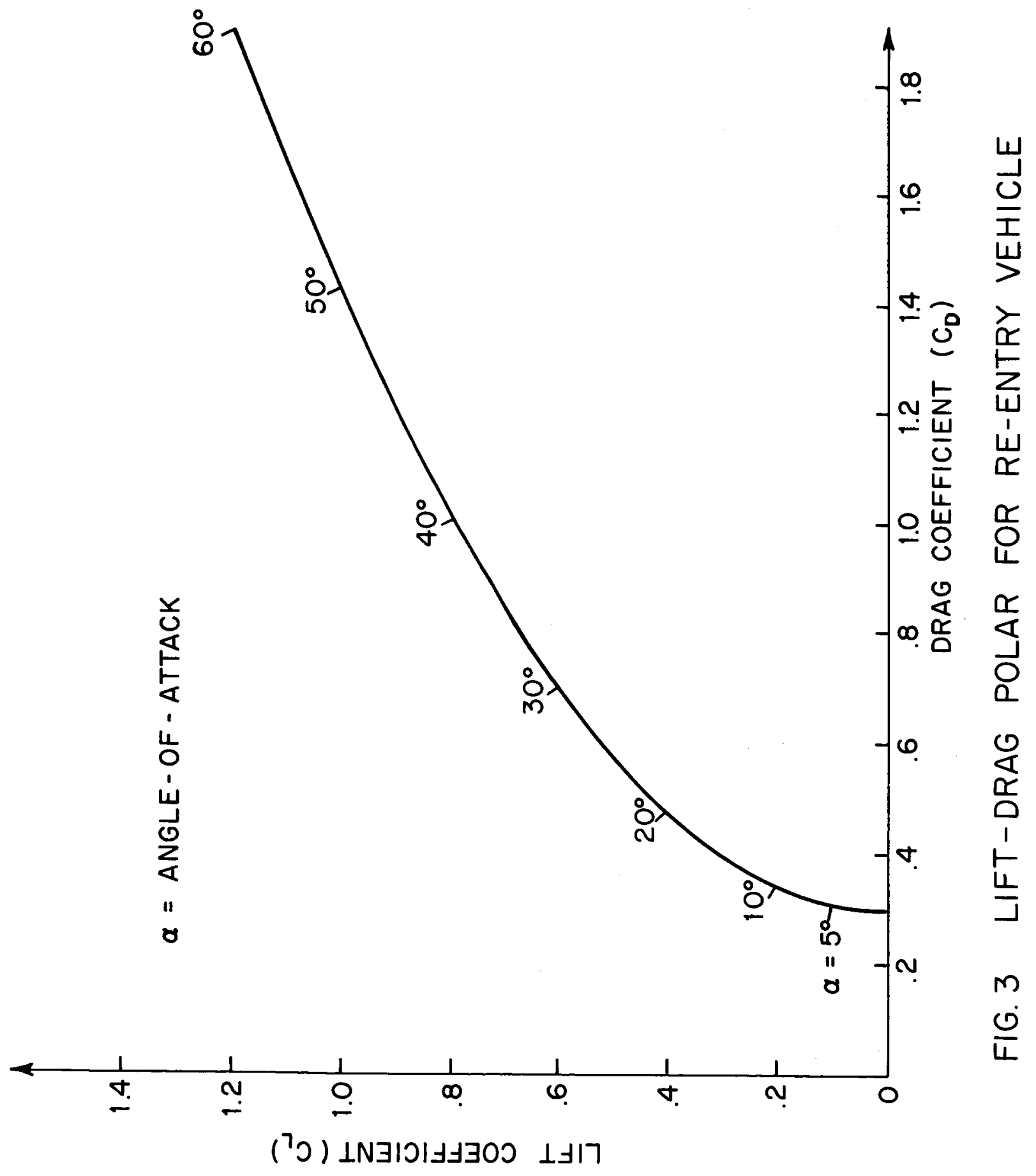


terminal condition on altitude and the inflight inequality constraint

$$
h(t) \leqslant h_{M}
$$

where $h_{M}$ is the given value of the maximum allowable altitude.

\section{SEPARATION OF ARCS FOR THE MAXIMUM RANGE PR OBLEM}

Starting from the initial conditions, a maximum range path eventually enters onto the constraint boundary at time $t_{1}$. At this point

$$
\begin{aligned}
& \mathrm{h}-\mathrm{h}_{M}=0 \\
& \stackrel{\mathrm{h}}{=} \mathrm{V} \sin \gamma=0
\end{aligned}
$$

must be satisfied as well as all along the constraint boundary. The control used to keep (20) and (21) satisfied on the constraint boundary is found from $\dot{\mathrm{h}}^{\circ}=0$ which implies

$$
C_{L}=\frac{2 m}{\rho_{M} v S}\left[\frac{g_{M}}{v}-\frac{v}{R+h_{M}}\right]
$$

where $\rho_{M}$ and $g_{M}$ are the values of $\rho$ and $g$ on the constraint boundary.

Since $h$ and $Y$ are fixed on the constraint boundary, only the velocity is free. The horizontal range travelled on the constraint boundary can be found as a function of the arc-entry and arc-exit velocities. The independent variable $t$ is eliminated by (15) so that

$$
R_{A}\left[t_{1}, t_{2}\right]=\int_{V\left(t_{2}\right)}^{V\left(t_{1}\right)} \frac{2 m d V}{\left(1+h_{M} / R\right) C_{D}{ }_{M} V S}=F\left[V\left(t_{1}\right)\right]-F\left[V\left(t_{2}\right)\right]
$$


where $C_{D}$ is a function only of velocity thraugh (22) and Fig. 2. Conceptually $R_{A}\left(t_{1}, t_{2}\right)$ depends only on the values of the exit and entry velocities although in general an analytic expression cannot be found.

Thus the problem can be reduced to two smaller problems in which the unconstrained arcs are found separately. The initial unconstrained arc from the initial conditions to the entry point onto the constraint boundary is found by obtaining a $\alpha(t)$ which maximizes,

$$
R_{I}=\int_{t_{0}}^{t} \frac{V \cos \gamma}{1+h / R} d t+F\left[V\left(t_{1}\right)\right]
$$

The terminal unconstrained arc from the exit point of the constrained arc to the terminal boundary is found by evaluating an $\alpha(t)$ which maximizes

$$
R_{F}=\int_{t_{2}}^{t_{f}} \frac{V \cos \gamma}{1+h / R} d t-F\left[V\left(t_{2}\right)\right]
$$

The sum of $R_{I}$ and $R_{F}$ is the total range $R_{A}$. One of the results of this optimization technique is to find the velocities at the two ends of the constrained arc。

If it is found that $V\left(t_{1}\right) \leqslant V\left(t_{2}\right)$ then no path of finite length lies on the constraint boundary although the optimal path may coincide with the constraint boundary at a point. In this case there is no separation. However, for a given set of constraint levels an intermediate point constraint must be imposed [2], defined as $S\left(x_{,} t_{1}\right)=0$. The Lagrange multiplier associated with the intermediate constraint must be positive (for maximization); if it is not an unconstrained path which lies below the constraint boundary will be better. 
-12 -

Necessary conditions for the two unconstrained arcs can be stated after first augmenting the performance indices as

$$
\begin{aligned}
& \bar{R}_{I}=\Phi_{I}+\int_{t_{0}}^{t}\left[H-\lambda_{v} \dot{V}-\lambda_{Y} \dot{Y}-\lambda_{h} \dot{h}\right] d t \\
& \bar{R}_{F}=\Phi_{F}+\int_{t_{2}}^{t_{f}}\left[H-\lambda_{v} \dot{V}-\lambda_{\gamma} \dot{\gamma}-\lambda \dot{h}\right] d t
\end{aligned}
$$

where

$$
\begin{aligned}
& \Phi_{I}=F\left[V\left(t_{1}\right)\right]+\nu_{h}\left[h\left(t_{1}\right)-h_{M}\right]+\nu_{\gamma} \gamma\left(t_{1}\right) \\
& \Phi_{F}=-F\left[V\left(t_{2}\right)\right]+\bar{\nu}_{h}\left[h\left(t_{f}\right)-h_{f}\right]
\end{aligned}
$$

and the variational Hamiltonian is

$$
\begin{aligned}
H= & \frac{V \cos \gamma}{1+h / R}-\lambda_{V}\left[\frac{C_{D^{\rho V^{2} S}}}{2 m}+g \sin \gamma\right] \\
& +\lambda_{Y}\left[\frac{C_{L} \rho V S}{2 m}+\left(\frac{V}{R+h}-\frac{g}{V}\right) \cos \gamma\right]+\lambda_{h} V \sin \gamma
\end{aligned}
$$

Here $\lambda_{v}, \lambda_{Y}, \lambda_{h}, \nu_{h}, \nu_{\gamma}, \bar{\nu}_{h}$ are Lagrange multipliers. The Euler-Lagrange equations are defined from (28) as

$$
\dot{\lambda}_{v}=-H_{v}, \dot{\lambda}_{Y}=-H_{\gamma^{*}} \dot{\lambda}_{h}=-H_{h}
$$

The boundary conditions for the initial unconstrained problem at $t_{1}$ are

$$
\lambda_{v}\left(t_{1}\right)=\Phi_{v\left(t_{1}\right)}=\left.\frac{2 m}{\left(1+\frac{h_{M}}{R}\right) C_{D^{\rho} M^{V S}}}\right|_{t=t_{1}}, \lambda_{\gamma}\left(t_{1}\right)=\Phi_{\gamma\left(t_{1}\right)}=\nu_{\gamma} \lambda_{h}\left(t_{1}\right)=\Phi_{h\left(t_{1}\right)}=\nu_{h}
$$


The boundary conditions for the terminal unconstrained problem at $t_{f} \operatorname{are}$

$$
\lambda_{v}\left(t_{f}\right)=\Phi_{v\left(t_{f}\right)}=0, \lambda_{Y}\left(t_{f}\right)=\Phi_{Y\left(t_{f}\right)}=0, \lambda_{h}\left(t_{f}\right)=\Phi_{h\left(t_{f}\right)}=\bar{\nu}_{h}
$$

while at the exit corner

$$
\lambda_{v\left(t_{2}\right)}=\Phi_{v\left(t_{2}\right)}=\left.\frac{2 \mathrm{~m}}{\left(1+\frac{h_{M}}{R}\right)\left(C_{D}^{\rho} M^{v S}\right)}\right|_{t=t_{2}}
$$

The original problem has been reduced to two, two-point boundaryvalue problems. For the initial arc the form of $h, \gamma$, and $\lambda_{v}$ are known at the entry point and the initial conditions are given. For the terminal arc

the form of $h, \gamma$, and $\lambda_{v}$, are known at the exit point whereas at the terminal boundary the values of $\lambda_{v}, \lambda_{Y}$ and $h$ are known. Note that the problem is time-independent. This implies that $\mathrm{H}=0$ all along the optimum path.

In this example there are three state variables and a second order SVIC. Since the problem is time-independent $n-1=p$.

\section{CALCULATION OF THE PERFORMANCE INDEX ON THE CONSTRAINED ARC}

An analytic expression cannot in general be found for the range when on the constraint boundary (23). However, when a successive improvement optimization scheme is used, some indication as to the improvement of the performance index is necessary. It is suggested that a table be made of range as a function of velocity starting at the largest expected value of $V\left(t_{1}\right)$ 
and ending at the lowest expected value of $V\left(t_{2}\right)$. The performance indices of (24) and (25) are written as $R_{I}-F\left(V_{B}\right)$ and $R_{F}+F\left(V_{L}\right)$ where $V_{B}$ and $V_{L}$ are chosen values in which on every iteration $V\left(t_{1}\right)>V_{B}$ and $V_{L}>V\left(t_{2}\right)$. Evaluating $F\left(V\left(t_{1}\right)\right)-F\left(V_{B}\right)$ and $F\left(V_{L}\right)-F\left(V\left(t_{2}\right)\right)$ on the computer is reduced to a table look-up.

However, one important case where an analytic expression can be found for (23) is for the lift idrag polar defined as

$$
\begin{aligned}
& C_{L}=C_{L_{0}} \alpha \\
& C_{D}=C_{D_{0}}+C_{D_{1}} \alpha^{2}
\end{aligned}
$$

For values of the constants of $C_{L_{0}}=.020, C_{D_{0}}=.297, C_{D_{1}}=.451 \times 10^{-3}$ the lift-drag polar of Fig. 2 is obtained from. (33) and (34). $\alpha(t)$ on the constraint boundary is now simply obtained from (33) and (22) as

$$
\alpha=\frac{2 m}{C_{L_{0}}^{\rho} M^{S}}\left(\frac{g_{M}}{V^{2}}-\frac{1}{R+h_{M}}\right)
$$

The drag coefficient of (34) is a function of velocity on the constraint

$$
C_{D}=C_{D_{0}}+C_{D_{1}}\left[\frac{2 m}{C_{L_{0}}^{\rho} M^{S}}\right]^{2}\left[\frac{g_{M}^{2}}{V^{4}}-\frac{2 g_{M}}{\left(R+h_{M}\right) V^{2}}+\frac{1}{(R+h)^{2}}\right]
$$

The analytic expression for the range on the constraint boundary solved by integrating (23) analytically is

$$
\begin{aligned}
& \mathrm{R}_{A}\left[t_{1}, t_{2}\right]=F\left[V\left(t_{1}\right)\right]-F\left[V\left(t_{2}\right)\right]= \\
& \frac{R_{1}}{2}\left[\frac{1}{2 Q_{2}} \log \left(Q_{2} V^{4}+Q_{4} V^{2}+Q_{5}\right)-\frac{Q_{4}}{Q_{2} Q_{6}} \tan ^{-1} \frac{2 Q_{2} V^{2}+Q_{4}}{Q_{6}}\right]
\end{aligned}
$$


where

$$
\begin{aligned}
& Q_{1}=\frac{2 m}{\left(R+h_{M}\right) \rho_{M} S}, Q_{2}=C_{D_{0}}+\frac{C_{D_{1}}}{C_{L_{0}}^{2}} Q_{1}^{2}, Q_{3}=\frac{\left.C_{D_{M}} g^{\left(R+h_{M}\right.}\right)}{C_{L_{0}}} \\
& Q_{4}=-\frac{2 Q_{3} Q_{1}^{2}}{C_{L_{0}}}, Q_{5} * \frac{\left[Q_{3} Q_{1}\right]^{2}}{C_{D_{1}}}, Q_{0}=\left(\frac{C_{D_{0}}}{C_{D_{1}}}\right)^{\frac{1}{2}} Q_{3} Q_{1}
\end{aligned}
$$

\section{RE}

For practical reasons, the re-entry problem may be complicated further by additional constraints. One such constraint is a limit on the resultant aerodynamic force. The ratio of the resulting aerodynamic force to the sea level weight is defined here as the number of g's,

$$
N_{g} \equiv \frac{\sqrt{L^{2}+D^{2}}}{m g_{0}}
$$

If $\mathrm{N}_{\mathrm{g}}$ is required to be less than some given number, this imposes a control variable inequality constraint on the trajectory. This constraint can be handled by the techniques of reference 2. It presents no obstacle to the separation of arcs as long as g-limit is always satisfied along the constraint boundary. Another practical constraint is a limit on the total heat absorbed by the heat shield. If the total heating is constrained the arcs cannot be separated in the maximum range problem with an altitude constraint. The amount of heat absorbed on one arc determines the amount of heat that can be absorbed on the other arc. The arcs are now dependent upon each other and the more complicated technique of reference 5 can be used. However, an alternative 
approach is to perform a parameter search on an equivalent problem that does separate. The heating rate is

$$
\dot{q}=C_{q} p^{\frac{1}{2}} v^{3}
$$

where $\mathrm{q}$ is the heat and $\mathrm{C}_{\mathrm{q}}$ is a known constant. A composite performance index can be formed using (39) and (18) as

$$
R_{q}=\int_{t_{0}}^{t_{f}}\left[\frac{v \cos \psi}{1+h / R}-K C_{q} \rho^{\frac{t}{z}} v^{3}\right] d t
$$

The procedure for finding optimal paths with a heating constraint is as follows: Choose a value for K. Since the problem is separable, the optimal arcs can easily be found and the total heat evaluated. If the value of total heating is greater than the desired value, $\mathrm{K}$ is increased; if less than the desired value, $K$ is decreased. For a new value of $K$ the optimal arcs and the total heating are again evaluated. This search for the proper value of $K$ is continued until the desired value of total heating is attained.

In general, integral constraints (the heating constraint above is an example) may be handled by this procedure.

\section{NUMERICAL DETERMINATION OF MAXIMUM RANGE}

\section{TRAJECTOR IES}

Numerical Methods The "Conjugate Gradient Method" of reference 4, was used to determine the two unconstrained arcs of the re-entry problem.

To check the results of the Conjugate Gradient Method a second order optimization program, the "successive sweep method" of references 6 and 8 , was used. This latter algorithm generates a sequence of improving paths 
by maximizing a quadratic approximation to the performance index.

Intitial Arc The initial conditions for this arc were taken as: V = 33, $961 \mathrm{ft} . / \mathrm{sec}$. , $\gamma=-1.57 \mathrm{deg}$, , and $h=189,890 \mathrm{ft}$. The terminal conditions at the entry point $f\left(t=t_{1}\right)$ onto the constraint boundary are $h=220,000 \mathrm{ft}$. and $\gamma=0\left(V\right.$ and $t_{1}$ are unspecified). The equations of motion were integrated forward from the given initial conditions until $\gamma$ becomes zero for the second time. In the conjugate gradient method the altitude constraint at the end of the arc was met using a quadratic penalty function. At that point $\lambda_{\gamma}$ was determined by setting the Hamiltonian equal to zero. Convergence was achieved in seven iterations using less than 15 seconds per iteration on the IBM 7094 computer. Fig. 4 shows, in altitude-range space, the starting nominal and some of the following iterations.

However, the trajectory of Fig. 4 is not the optimum path; it is only a local optimum. Fig. 5 shows this path with another locally optimum path that gives $30 \%$ more range for the initial arc down to a velocity of 26,494 $\mathrm{ft}$. /sec (from this velocity on, the maximum range paths are the same). The increase in range over the entire flight is $6 \%$. The existence of two locally optimal paths was not detected in either reference 11 or 5.

These two paths arise from widely different control strategies (See Fig. 7)。 Path 2 in Fig。 5, 6, and 7 uses low angles-of-attack to keep the drag small and consequently penetrates deeply into the atmosphere where air density is high. Path 1 uses larger values of angle-of-attack to keep the vehicle at higher altitudes where air density and drag are lower. Path 1 seems to concentrate on maximizing $F\left[V\left(t_{1}\right)\right]$ in Eqn. (24) whereas path 2 seems to 


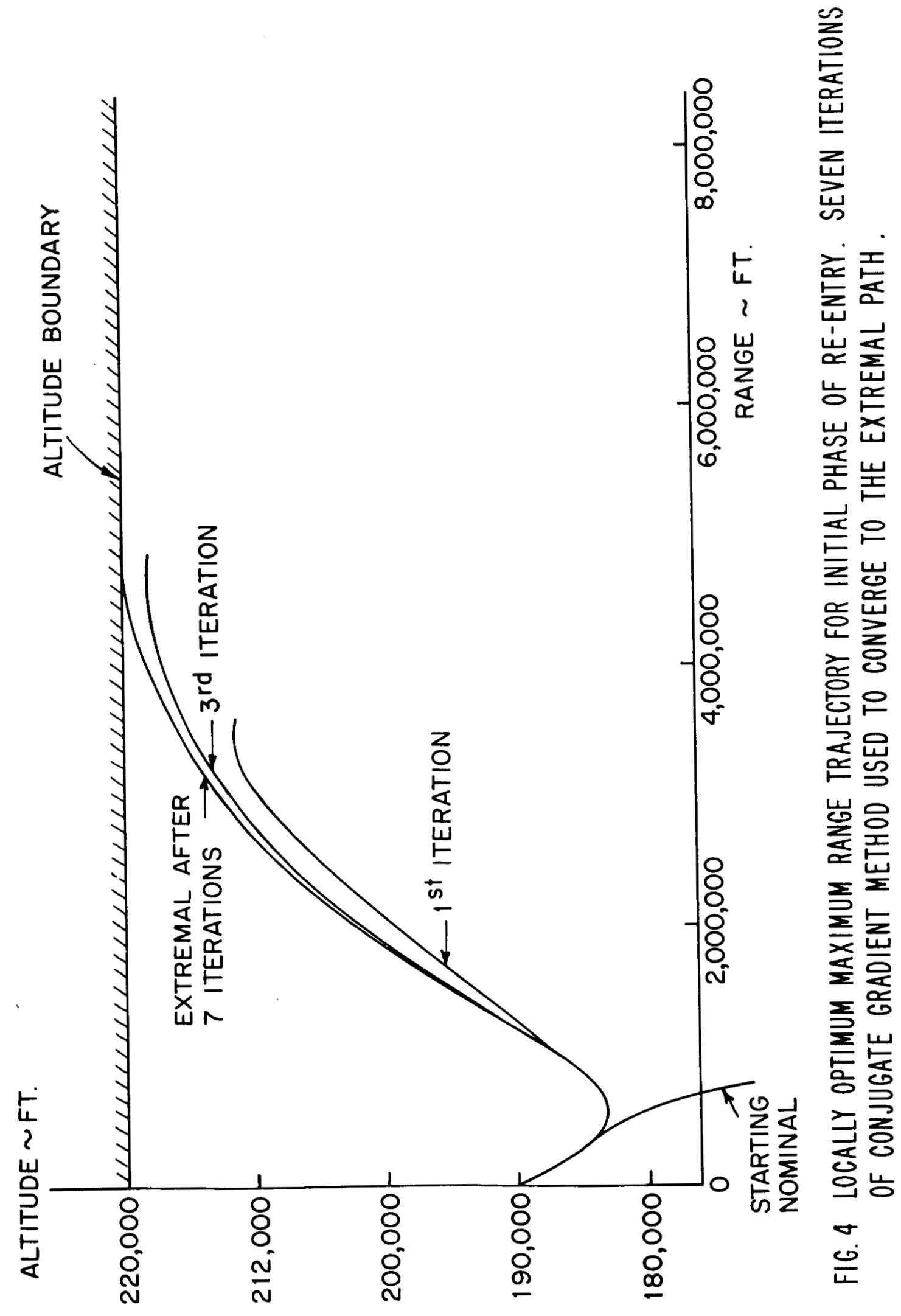




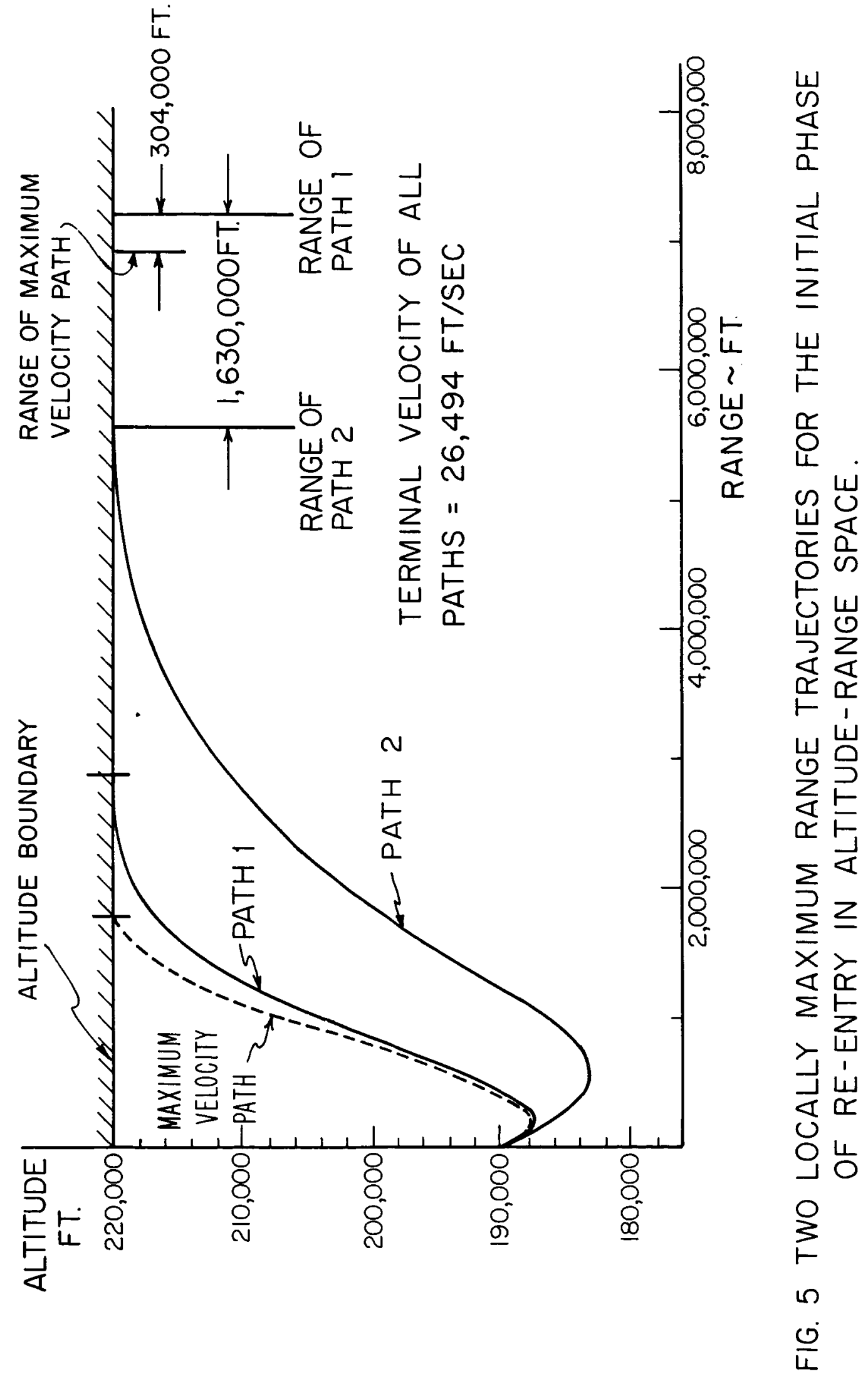




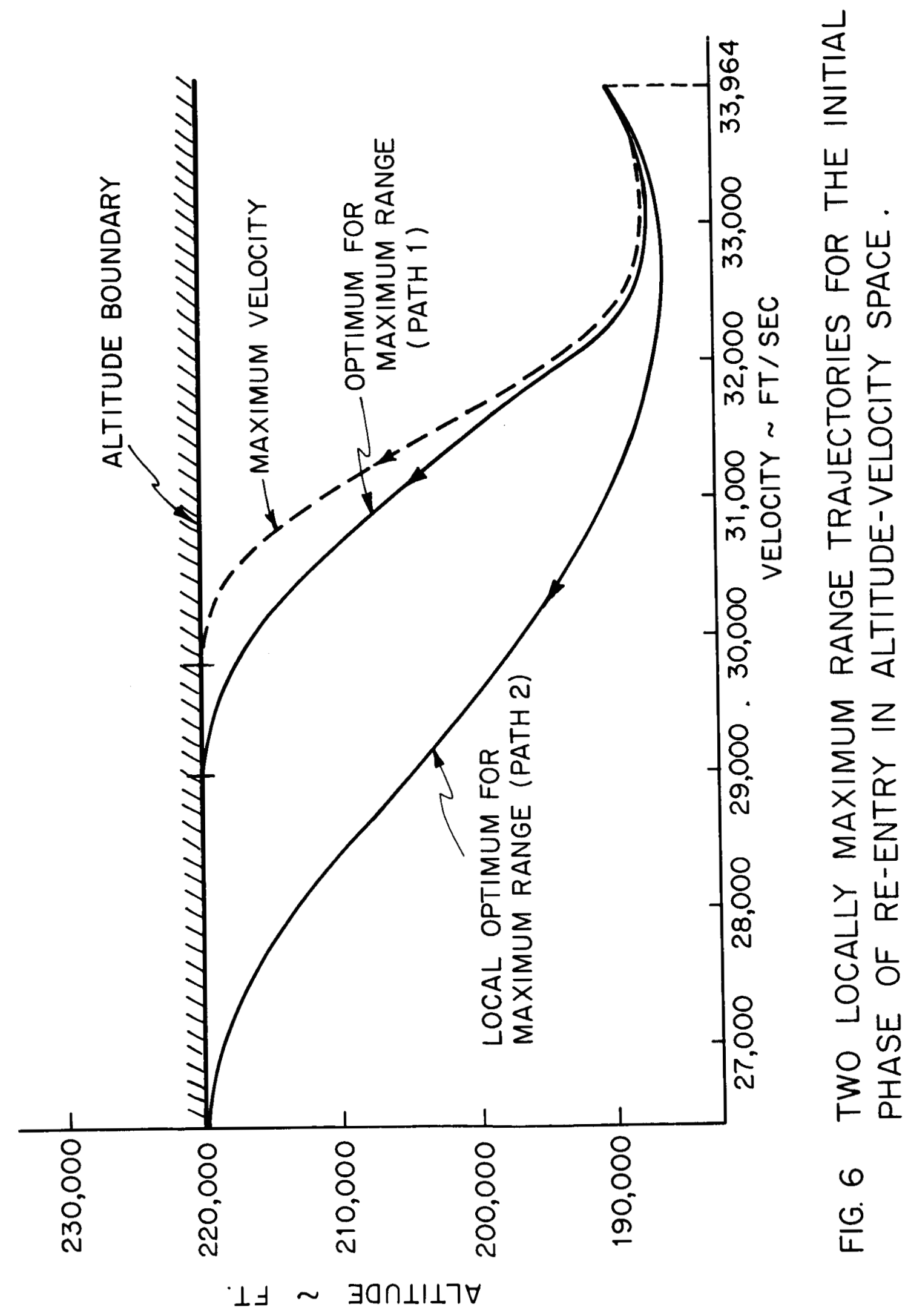




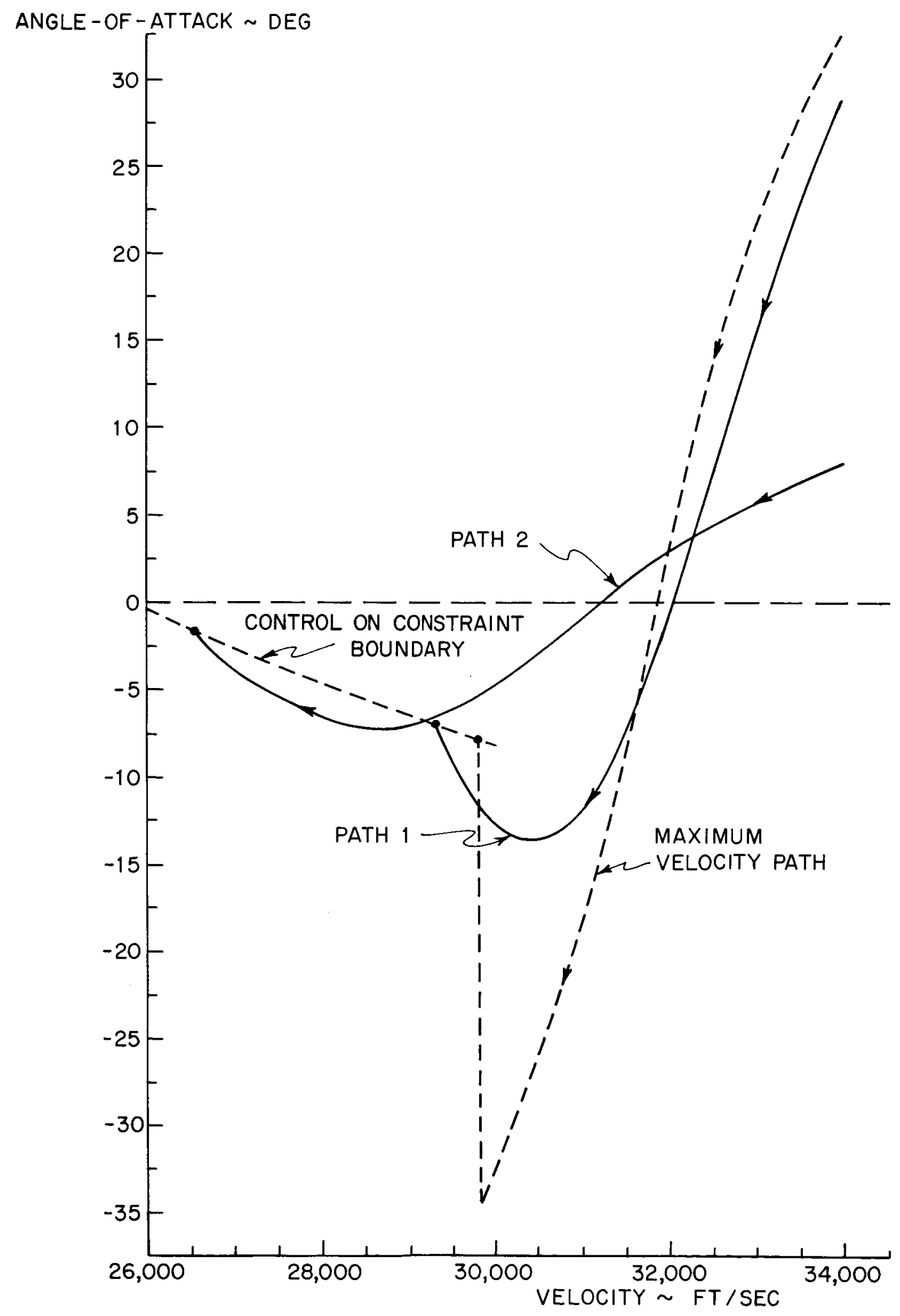

FIG. 7 ANGLE - OF-ATTACK Vs. VELOCITY FOR TWO LOCALLY MAXIMUM RANGE PATHS. 
concentrate on maximizing the integral (the range) in Eqn (24). Path 1 and 2 are shown in Fig. 6 in altitude-velocity space.

Continuity of the $\alpha$-Program Results obtained in reference 5 show a discontinuity in the $\alpha$-program at the entry point onto the constrained arc.

The control should be continuous since the variational Hamiltonian is regular [11]. The performance index is not very sensitive to this discontinuity so first order methods have great difficulty in obtaining continuous $\alpha$-programs. The second order scheme demonstrates clearly that $\alpha$ is continuous across the entry point for path 1 .

Maximum Velocity Path The trade off between entry point velocity and range in the performance index suggests that the maximum velocity path may be a good approximation to the maximum range path. The maximum velocity path is shown by a dashed path in Figs, 5,6, and 7. The maximum velocity path (Fig. 5) plus the constrained path down to $26,494 \mathrm{ft} / \mathrm{sec}$. gives only $5.5 \%$ less range than path 1 and $24.5 \%$ more range than path 2 . Initially, the angle-ofattack program for maximum velocity resembles that of path $1,($ Fig. 7 ), however, as the paths near the entry point, $\alpha$ for path 1 bends over. The difference in velocity at the entry point between the maximum velocity path and path 1 is $520 \mathrm{ft} / \mathrm{sec}$. as seen in Fig. 6 .

Conjugate Point First-order computing methods try to improve performance index on each iteration, without concern for the change in the size of the gradient. They will not converge to an extremal path that contains a conjugate point, since such a path is not an optimal path. 
An attempt was made, using the second-order sweep method to check the results obtained for path 2 of Fig. 5 by the first-order conjugate gradient method. However, all attempts at solution of the matrix Riccati equation (which governs the second partial derivatives of the optimal return function with respect to the state variables) resulted in overflow of the computer $\left(10^{38}\right)$. This led us to suspect the presence of conjugate points in the vicinity of the extremal field for the following reasons:

(a) Using the conjugate gradient method to solve the maximum range problem, both the performance index and the norm of the gradient increased for some iterations, This behavior indicates that a conjugate point might exist.

(b) The sweep method tries to decrease the magnitude of the gradient on each iteration, without concern for the change in the performance index. Hence, the method may very well move toward an extremal path containing a conjugate point; however, convergence to such a path will not be obtained, because solutions to the Ricatti equation, as mentioned above, will overflow the computer first.

(c) A necessary (but not sufficient) condition for the existence of a conjugate point on an extremal path in a maximization problem is for the matrix

$$
\mathrm{B} \triangleq \mathrm{H}_{\mathrm{xx}}-\mathrm{H}_{\mathrm{x} \alpha} \mathrm{H}_{\alpha \alpha}{ }^{-1} \mathrm{H}_{\alpha \mathbf{x}}
$$

to have some positive eigenvalues over all or part of the path (cf. refs. 7 and 9). If $B$ is negative-definite over the whole path there can be no conjugate points. For both paths 1 and 2 in Fig. 5 we found that $B$ did indeed have some positive eigenvalues. 
Terminal Arc At neither end of the terminal arc are all the state variables specified. In the conjugate gradient method the missing initial conditions are treated as control parameters chosen to maximize the objective function. At the initial point of the terminal arc $h$ and $Y$ are known but $V$ is to be determined. From (25) and (32)

$$
\left.\frac{\partial R_{F}}{\partial V}\right|_{t=t_{2}}=\lambda_{v}\left(t_{2}\right)-\frac{1}{\left(1+\frac{h_{M}}{R}\right)\left(\frac{C_{D} \rho_{M} V S}{2 m}\right)}
$$

The optimization process drives $\frac{\partial R_{F}}{\partial \gamma_{r}}$ to zero making $\lambda_{v}\left(t_{2}\right)$ equal to the required value.

The optimal path obtained is shown in Fig. 8 in the altitude-range space. The $\alpha$-program corresponds very closely to the $\alpha$ for maximum L/D (lift over-drag ratio) except near the terminal point where high values of angle-of-attack are used in the flare-out maneuver. Fig. 9 shows the $\alpha$ history as a function of range. The exit velocity determined by a parameter search is $19,010 \mathrm{ft} / \mathrm{sec}$.

\section{CONCLUSIONS}

A sufficient condition for separate computation of arcs for certain optimization problems with state variable inequality constraints was formally presented. This concept was applied to the problem of maximizing the range of a glider entering the Earth's atmosphere at parabolic speeds subject to a maximum altitude constraint after the initial pull up. In numerically determining the unconstrained arcs, the conjugate gradient method converged extremely rapidly. This allowed a detailed investigation of maximum range 


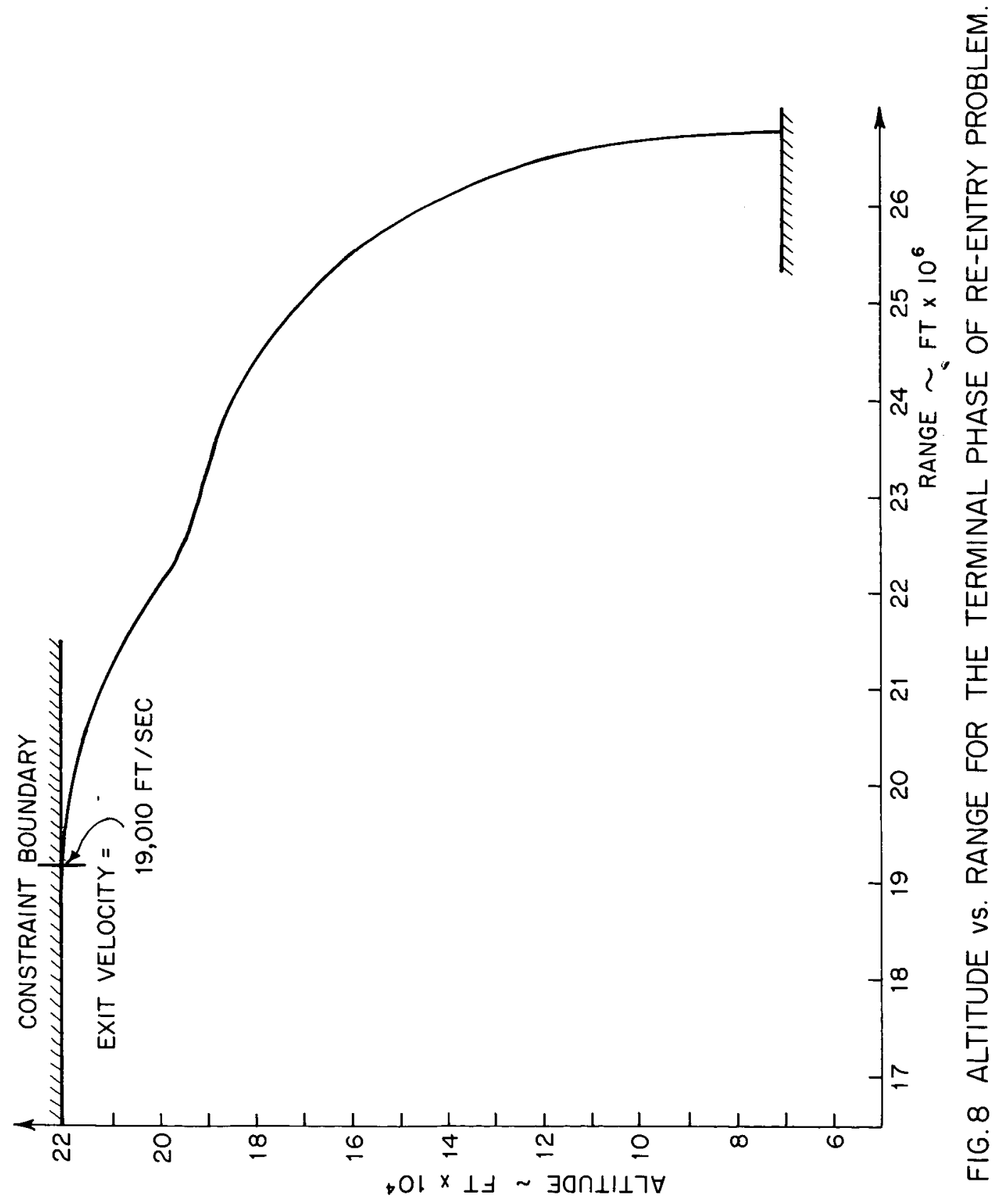




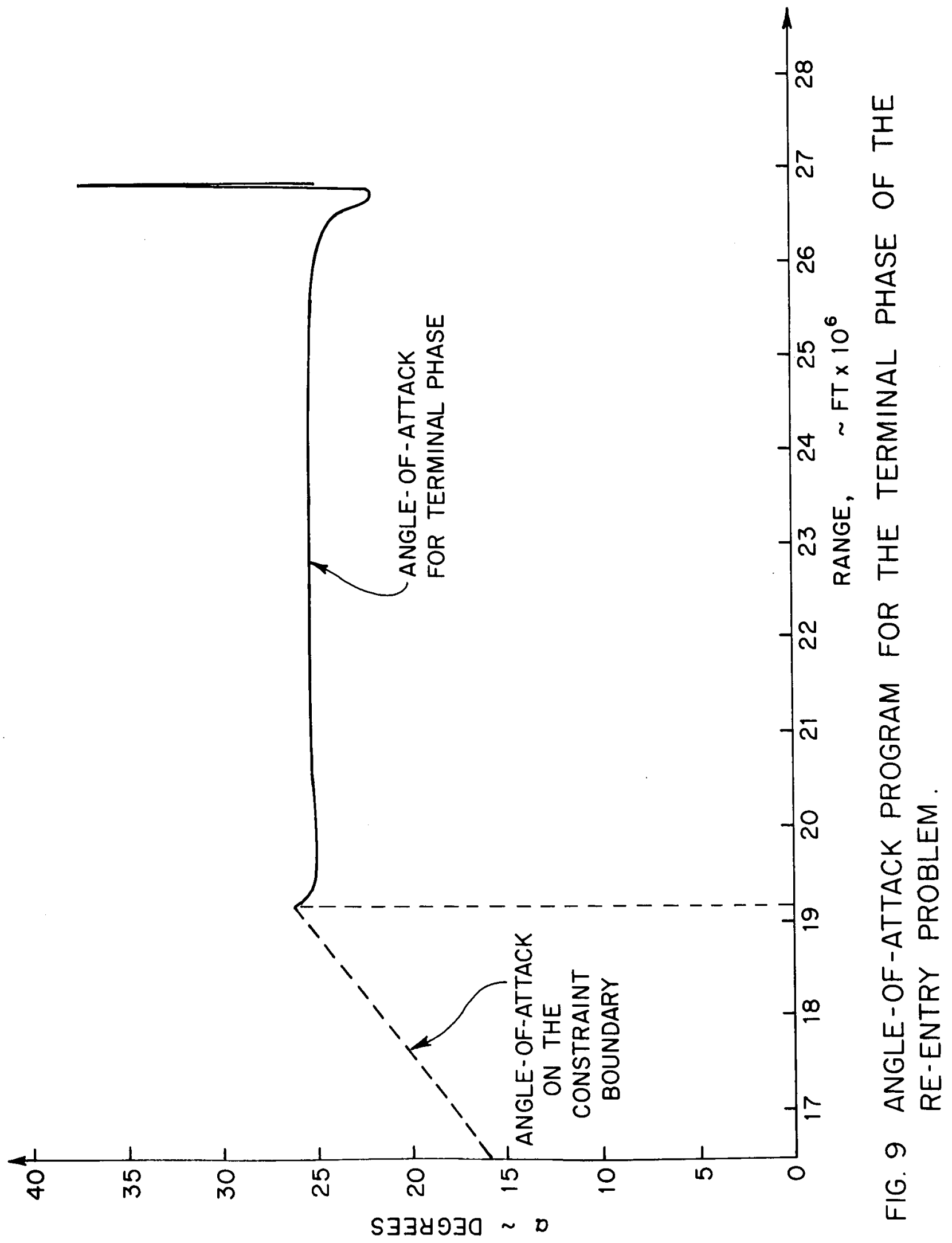




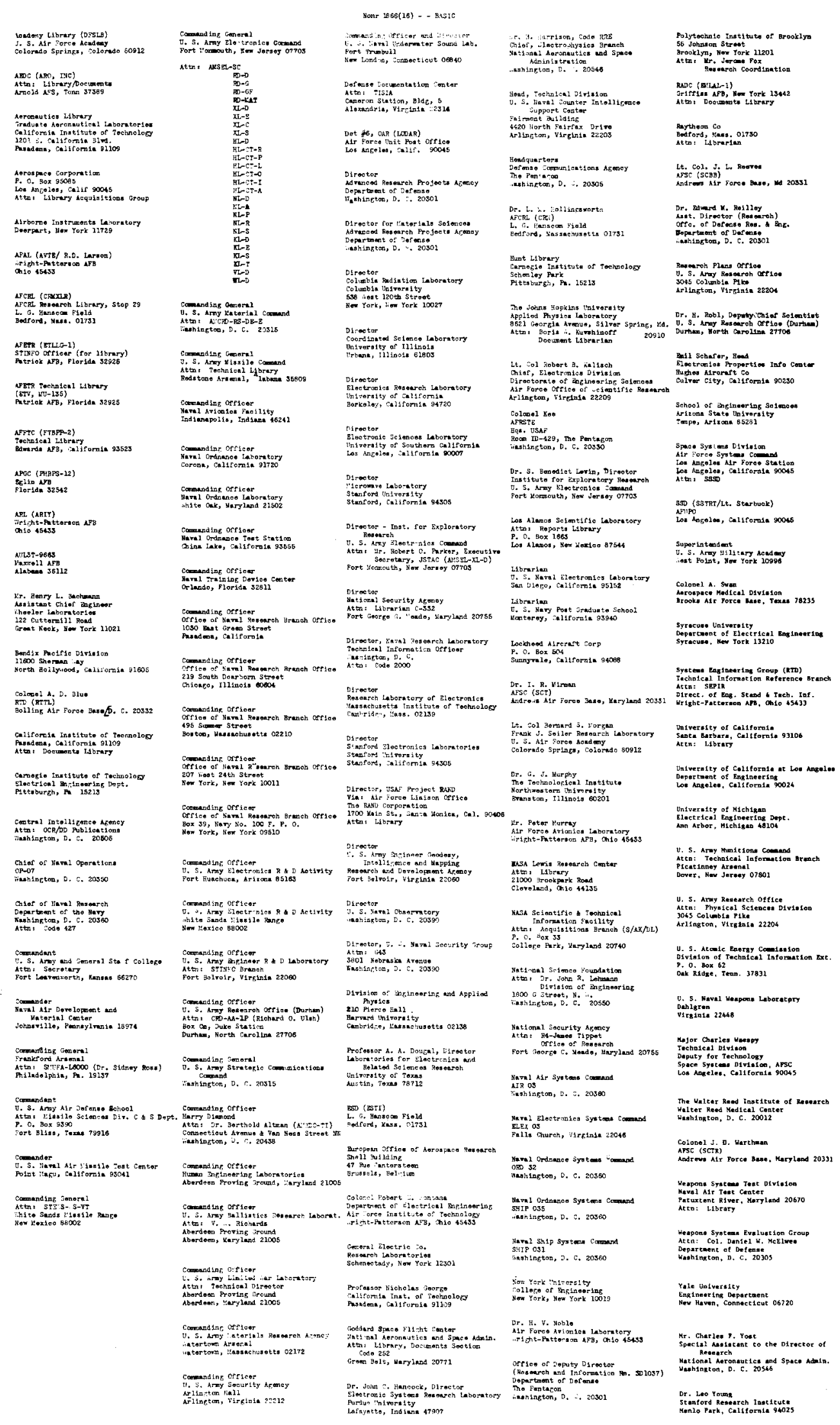


trajectories.

For the initial phase of re-entry two locally maximum range arcs were found. This appears to be a consequence of the lift-drag characteristics of the vehicle and the decrease in air density with altitude. Both first and second order methods indicate a conjugate point behavior in the initial phase extremal field.

\section{REFER ENCES}

1. Gamkrelidze, R. V. "Optimal Processes with Bounded Phase Coordinates," Izv. Akad. Nauk. USSR, Sec。Mat., Vol。24, pp.315-356, 1960

2. Bryson, A. E. Jr。, Denham, W. F., Dreyfus, S. E., "Optimal Programming Problems with Inequality Constraints I: Necessary Conditions for Extremal Solutions," AIAA Journal, Vol. 1, No. 11, November, 1963.

3. Kelley, $\mathrm{H}$ 。 J. "Method of Gradients," $\mathrm{Ch} .6$ of Optimization Techniques, edited by G. Leitmann, Academic Press, New York, N. Y., 1962.

4. Lasdon, L. S., Mitter, S. K., Warren, A. D., "The Method of Conjugate Gradient for Optimal Control Problems," Proc. IEEE, p. 904, June 1966.

5. Denham, W. F., Bryson, A. E。 Jr., "Optimal Programming Problems with Inequaltiy Constraints II: Solution by Steepest-Ascent" AIAA Journal Vol。2, No。 1, January 1964。

6. McReynolds, S. and Bryson, A.E.Jr., "A Successive Sweep Method for Solving Optimal Programming Problems, "Sixth Joint Automatic Control Conference, Troy, New York, June 1965.

7. Breakwel1, J. V. and Ho, Y. C., "On the Conjugate Point Condition for the Control Problem," Int. Journal of Engineering, Vol.2, pp.565-579, 1965.

8. Mitter, S. K., "Successive Approximation Methods for the Solution of Optimal Control Problems," Automatica, Vol. 3, pp。135-149, 1966.

9. Bryson, A. E. Jr。, and Ho, Y. C., "Optimization, Estimation, and Control," Lecture Notes, Harvard University, 1966-1967.

10. Paiewonsky, Bernard, "On Optimal Control with Bounded State Variables," Aeronautical Research Associates of Princeton, Inc. Report No. 60. July 1964.

11. Lasdon, Warren, and Rice, "An Interior Penalty Function Method for Inequality Constrained Optimal Control Problem, "Case Institute of Technology, Cleveland, Ohio, November 1966. 


\section{DOCUMENT CONTROL DATA - R \& D}

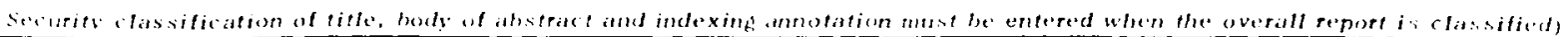
G TING ACTIVITY (Corporate atthor)

Division of Engineering and Applied Physics

Harvard University Cambridge, Massachusetts 2a. REFOFI SECURITY CLACSIIICATION Unclassified 2h. GROUP

REPORT TITLE

THE SEPARATE COMPUTATION OF ARCS FOR OPTIMAL FLIGHT PATHS WITH STATE VARIABLE INEQUALITY CONSTRAINTS

4. DESCRIPTIVE NOTES (Type of report and,inclusive dates)

Interim technical report

5. AUTHOR(S) (First name, middle initial, last name)

Jason L. Speyer, Raman K. Mehra, and Arthur E. Bryson, Jr.

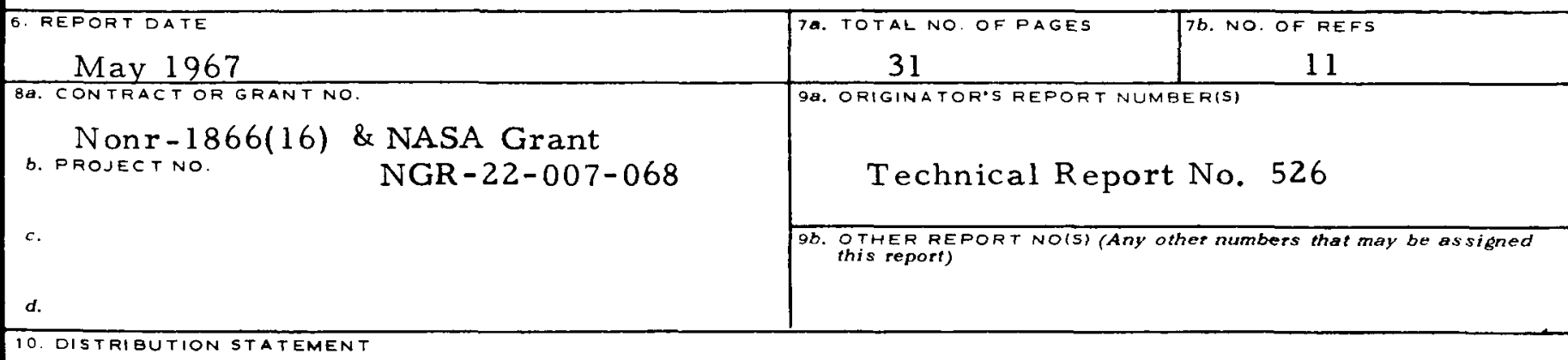

$R$ eproduction in whole or in part is permitted by the $U$. S

Government. Distribution of this document is unlimited.

\begin{tabular}{|l|l|}
\hline 11. SUPPLEMEN TARY NOTES & i2. SPONSORING MILITARY ACTIVITY \\
Office of Naval Research
\end{tabular}

Separate computation of arcs is possible for a large class of optimization problems with state variable inequality constraints. Surprisingly, this class (to the best of the authors' knowledge) includes all physical problems which have been solved analytically or numerically to date. Typically thse problems have only one constrained arc. Even in more complex problems, separation of arcs can be used to search for additional constrained arcs.

As an important example, a maximum range trajectory for a glider entering the Earth's atmosphere at a supercircular velocity is determined, subject to a maximum altitude constraint after initial pull-up. It is shown that the optimal path can be divided into three arcs, which may be determined separately with no approximations. The three arcs are (1) the initial arc, beginning at specified initial condition and ending at the entry point onto the altitude constraint; (2) the arc lying on the altitude constraint; and (3) the terminal arc, beginning at the exit point of the altitude constraint and ending at some specified terminal altitude.

The conjugate gradient method, (ref. 4), a first order optimization scheme is shown to converge very rapidly to the individual unconstrained optimal arcs. Using this optimization scheme and taking advantage of the separation of arcs an investigation revealed that two locally optimum paths exist. The range of one exceeds the range of the other by about 250 nautical miles (about 6\%) for the re-entry vehicle used here (maximum lift-to-drag ratio is .9). 


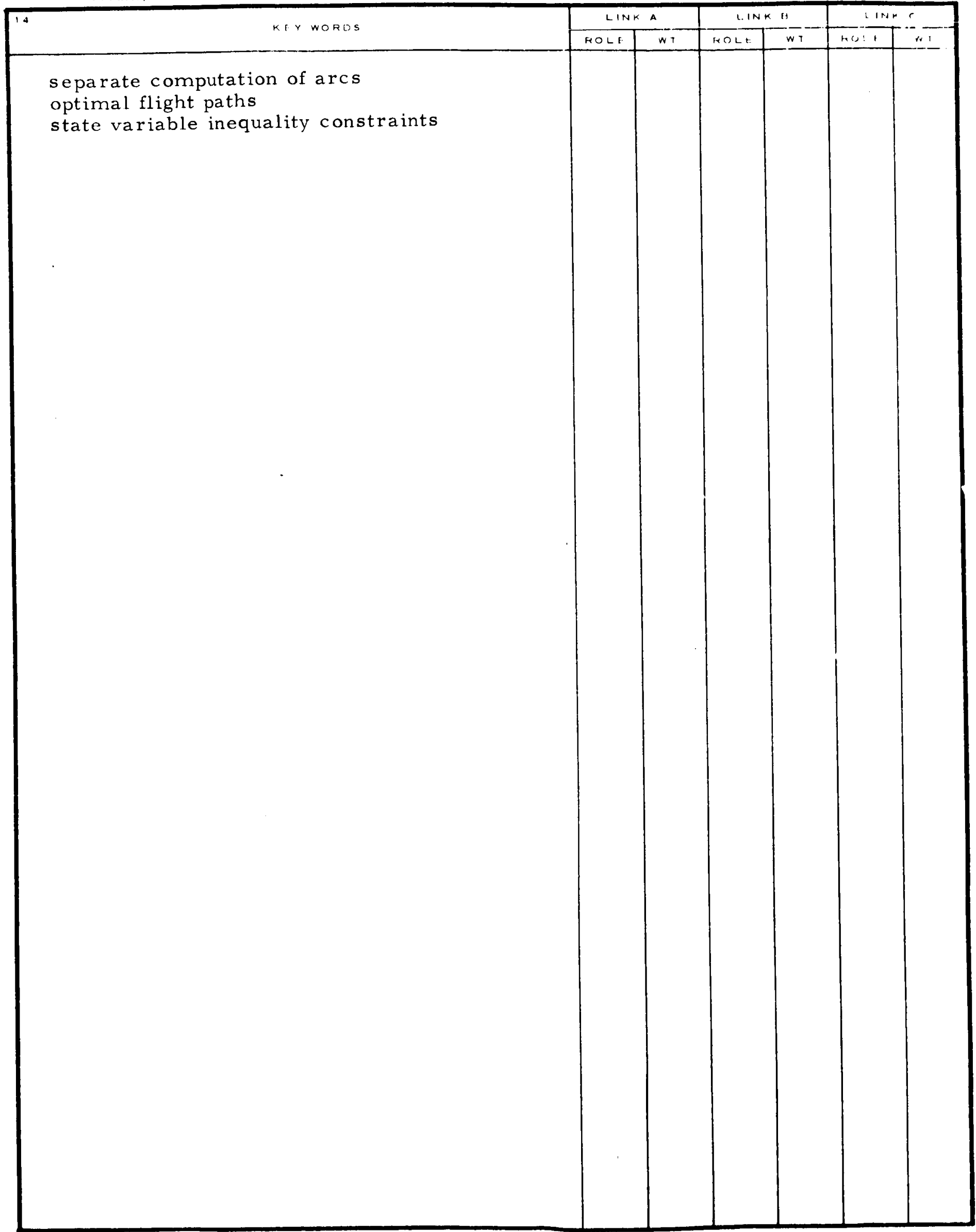

